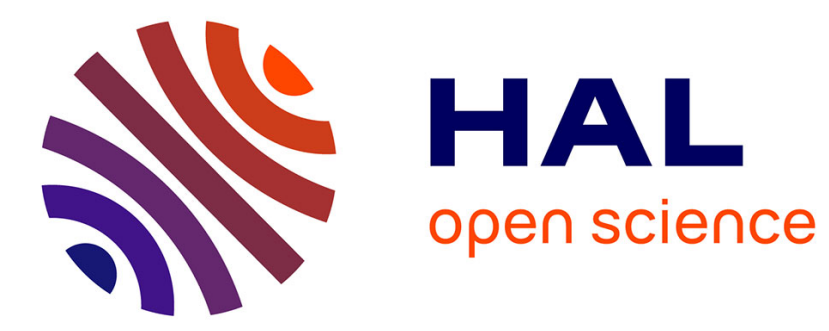

\title{
Cooperative guidance laws for maneuvering target interceptions
}

Hélène Piet-Lahanier, Arthur Kahn, Julien Marzat

\section{To cite this version:}

Hélène Piet-Lahanier, Arthur Kahn, Julien Marzat. Cooperative guidance laws for maneuvering target interceptions. 19th IFAC Symposium on Automatic Control in Aerospace (ACA 2013), Sep 2013, Würzburg, Germany. pp.301-306. hal-00861972

\section{HAL Id: hal-00861972 https://hal.science/hal-00861972}

Submitted on 14 Sep 2013

HAL is a multi-disciplinary open access archive for the deposit and dissemination of scientific research documents, whether they are published or not. The documents may come from teaching and research institutions in France or abroad, or from public or private research centers.
L'archive ouverte pluridisciplinaire HAL, est destinée au dépôt et à la diffusion de documents scientifiques de niveau recherche, publiés ou non, émanant des établissements d'enseignement et de recherche français ou étrangers, des laboratoires publics ou privés. 


\title{
Cooperative guidance laws for maneuvering target interceptions
}

\author{
H. Piet-Lahanier* A. Kahn* J. Marzat* \\ * ONERA - The French Aerospace Lab, F-91123 Palaiseau, France \\ \{helene.piet-lahanier, arthur.kahn, julien.marzat\}@onera.fr
}

\begin{abstract}
This study addresses the design of cooperative guidance laws for interceptor missiles. A polyhedric bounded-error estimation of the target position is employed, based only on the assumption that the target acceleration and measurement noise remain within known bounds. This prediction of the maneuvering target trajectory is then incorporated into a model predictive control scheme to derive the missile guidance law. The cases of both single and multiple targets are investigated. Simulation results are presented to illustrate the proposed approach.
\end{abstract}

Keywords: cooperation, guidance law design, maneuvering targets, model predictive control, set-membership estimation.

\section{INTRODUCTION}

Most of the guidance laws designed for missile interception focus on one-to-one scenarios. However the development of very agile targets can be very demanding in terms of technological properties for a single interceptor. In order to lower the cost induced by the development of such abilities on a single vehicle, research has been focused on how this complex mission could be effectively fulfilled using a large number of cheaper less-sophisticated vehicles. However, to provide the same result as the one sought for initially, all the vehicles must cooperate so that the flock obtains performances at least equal but possibly greater than those of a complex system (Gillen and Jacques (2002)). Cooperation requires to split the initial task in several subtasks and affect each subtask to a specific vehicle while coordination of the entire vehicle set must guarantee the satisfaction of the initial mission needs. The problem is now to determine the dynamic behavior of each vehicle. It must satisfy the following points:

- Individual mission combination must provide the same expected result as the initial complex mission

- Each trajectory must ensure a secure flight to the vehicle following it but also to the rest of the flock.

Since the late 1980s, research has been pursued in cooperative control of vehicles (Gerkey and Mataric (2002)). Depending on the type of mission, level of information, and safety requirements, various solutions have been presented. In Murray (2007), a survey of recent research in cooperative control of multi-vehicle systems is presented. Many applications in military and civil domains have been developed, including formation flights or swarms (Jadbabaie et al. (2003)), cooperative attack and rendez-vous (Chandler et al. (2001)), or wide area coverage (Ahmadzadeh et al. (2006)).

Approaches have been developed in Onera DCPS that ensure simultaneously collision avoidance and flock regulation (Rochefort et al. (2011)). Extension of these methods is currently studied for application to missile raid definition and wide area surveillance. In this paper, the description of a set of common hypotheses will be first presented. Derivation of set-membership target tracking and associated guidance laws for interception will be introduced. Application of such methods to missile raid will be presented.

\section{PROBLEM STATEMENT}

\subsection{Definitions}

The following definitions will be used in this article. A vehicle is described by a dynamical system whose state vector includes its position and velocity in the three dimensional space. The flock or raid consists in a set of $N$ vehicles. The vehicles are either able to communicate with each other or evaluate the predicted motion of other members of the flock or raid. The dynamics of the $i$ th vehicle is assumed to be described as

$$
\mathbf{x}_{k+1}^{i}=f^{i}\left(\mathbf{x}_{k}^{i}, \mathbf{u}_{k}^{i}\right)
$$

where $\mathbf{x}^{i}$ is the $i$ th state vector of dimension $n$, and $\mathbf{u}^{i}$ is the $i$ th control vector of dimension $m$. The role of a vehicle expresses the part of the mission to which it is devoted. Depending on the problem under consideration, the role of vehicle $i$ will be described by a function $g^{i}$

$$
g^{i}\left(\mathbf{x}^{i}, \mathbf{x}^{j}, \mathbf{y}\right), j=1, \ldots, N, j \neq i,
$$

where $\mathbf{y}$ is an information vector depending on the mission of variable dimension. The link between $\mathbf{u}^{i}$ and $g^{i}$ will be defined depending on the application considered, so that the vehicle fulfills its role among the flock. A general description of $\mathbf{u}^{i}$ is given by the relationship:

$$
\mathbf{u}^{i}=F^{i}\left(\mathbf{x}^{i}, g^{i}\left(\mathbf{x}^{i}, \mathbf{x}^{j}, \mathbf{y}\right)\right), j=1, \ldots, N, j \neq i,
$$

where $F^{i}$ is assumed to be a smooth vector-valued function. A performance criterion $J$ must be defined in order to qualify the global quality of the mission. As the vehicles collaborate, this criterion cannot be split straightforwardly into $N$ independent subfunctions associated to each vehicle. Each vehicle must dispose of its own criterion $J^{i}$ whose 
computation depends on its own state and measured or predicted states of the other vehicles, in a decentralized way.

\subsection{Cooperative guidance laws for interceptor missiles}

Optimization of engagement with cooperating defending missiles should increase the probability that at least one missile reaches the target. The definition of cooperative guidance laws for missile raids first relies on the definition of the cost function that must reflect the ability of the missile to intercept the target and select control laws that enhance the cooperation between the vehicles. The cooperative performance criterion must be designed in order to allocate efficiently the interceptor missiles towards potential target locations in order to prevent its escape. It must also ensure that the intercepting trajectories are safe and avoid collateral damages. Several approaches have been developed for cooperative attacks as in Rasmussen et al. (2004), Le Menec et al. (2011), Wei et al. (2008). The differences arise mostly on the means of prediction of target behavior and structure of the guidance laws.

In this paper, the target prediction is performed using setmembership estimation. It provides bounds of potential variations of the target state vector. The performance criterion is thus defined to maximize interception ability on the predicted bounded space where the target is located. It is completed with terms for control limitations and collateral damage avoidance. The elaboration of the corresponding guidance law for each interceptor missile is based on nonlinear model predictive control (MPC). This type of control law consists in determining a control input sequence that minimizes a cost function depending on the measurements, predicted states and control inputs up to a prediction horizon $H_{p}$ steps ahead, with $H_{p}$ fixed (Garcia et al. (1989)). The main advantage of MPC is thus to anticipate future consequences of a control input. The control-input sequence is optimized (over its admissible range) up to the control horizon $H_{c}$, with $H_{c} \leq H_{p}$, and assumed to remain constant during the following time steps between $H_{c}$ and $H_{p}$. The first sample of the optimal control sequence is applied at time $k$ up to the following observation time $k+1$. The use of MPC for cooperative guidance scheme allows to take into account the objectives of other vehicles and to deal with fulfillment of various aims by means of the cost function. Current research on cooperative distributed MPC has been presented to establish convergence (Dunbar and Murray (2004); Müller et al. (2011)), robustness (Siva and Maciejowski (2011)) and formation flying (Olfati-Saber et al. (2003)). Application to guidance of flock has been presented in Rochefort et al. (2012).

The resulting performances are illustrated on two scenarios. The first one considers two interceptor missiles aiming at a single target with potential maneuvering abilities. The second one considers the cooperative interception of two targets by four interceptors.

\section{ESTIMATION AND PREDICTION OF TARGET TRAJECTORY}

The difficulty of predicting target position at interception stems from the uncertainty in interception time. The efficiency of target maneuvers has greatly improved recently, lessening the gap between interceptor and target maneuverability. Accurate estimation of the target acceleration remains a tough problem although efficient interception would require increased performance in prediction of the target evolution.

\subsection{Target modeling and estimation}

The determination of maneuvering target trajectories relies mostly on the use of a state-space discrete model with additive noise of the form

$$
\begin{array}{r}
\mathbf{x}_{k+1}^{c}=f_{k}^{c}\left(\mathbf{x}_{k}^{c}, \mathbf{u}_{k}^{c}\right)+\mathbf{w}_{k}^{c}, \\
\mathbf{z}_{k}^{c}=h_{k}^{c}\left(\mathbf{x}_{k}^{c}\right)+\mathbf{v}_{k}^{c}
\end{array}
$$

where $\mathbf{x}_{k}^{c}, \mathbf{z}_{k}^{c}$ and $\mathbf{u}_{k}^{c}$ are the target state, observation and control input vectors, respectively, at the discrete time step $k$. Process and measurement noise sequences are represented by $\mathbf{w}_{k}^{c}$ and $\mathbf{v}_{k}^{c}$ respectively. Functions $f_{k}^{c}$ and $h_{k}^{c}$ are vector-valued functions selected for representing the assumed dynamics of the system. The measurements are provided either by a ground-based device with slow uplink frequency or by a missile-borne seeker with higher frequency. Estimation and prediction of the state vector are most often carried out with Kalman or extended Kalman filter (Singer and Behnke (1971)). However, the resulting prediction of the state vector is based on the mixture of a Gaussian evolution and a deterministic trajectory aimed at a given objective. The adequacy of the prediction is then tightly linked to the credibility of this assumption. The prediction of the target positions can also be performed using probability density function derived from statistical assumptions on the target potential maneuvers (Best and Norton (2000)). The derivation of the resulting probability density function associated with the target predicted locations may prove difficult to obtain.

\subsection{Determination of bounding sets}

When the target maneuvers, the missile has to select one among a variety of maneuver dependent interception positions within the range of interception time. The optimization of missile trajectory is then tightly linked to assumption on the potential interception locations of target at interception time. This is why it has been suggested in Shin et al. (2011) to describe the predicted target positions as a set whose boundaries depend on target noise, potential acceleration and potential objectives. This is based on the use of bounded-error approach. The dynamical model of the target is a simple discrete point trajectory with acceleration inputs. The sole assumption on the inputs and noise measurements is that they remain within given bounds. In this framework, the resulting estimate is a set whose boundaries have to be characterized. This corresponds to guaranteed estimation that was initially introduced by Schweppe (1968) and followed by Bertsekas and Rhodes (1971). Several methods have been developed to define the boundary of this set or to compute a set guaranteed to contain it. Most of the existing approaches compute a simple convex set such as an ellipsoid (Durieu et al. (2001)), but other methods have been described that result in characterizing a polyhedral boundary or determine the union of intervals to which all state vectors belong (Walter 
and Kieffer (2003)). The interest of polyhedral boundary in this context is that the characterization of the collision cone as defined in Chakravarthy and Ghose (2011) is directly derived from this description and could be used for the cooperative guidance laws.

The polyhedral boundary consists of a set of vertices and supporting hyperplanes. The determination of this set is performed using the following algorithm derived from Walter and Piet-Lahanier (1989) and illustrated in Figure 1 for a 3D state vector.

- From the initial target state (i.e. position and speed), set $N$ initial points corresponding to the vertices of a convex polyhedron surrounding the target state (red polyhedron)

- Update the set using new measurements if available by removing vertices that do not belong to the intersection of the polyhedron with the set of admissible measurements (blue polyhedron)

- At each time step, predict each vertex position in the state space using the mean input values

- Expand the resulting polyhedron using the bounds on the potential acceleration in the direction of set expansion (green polyhedron)

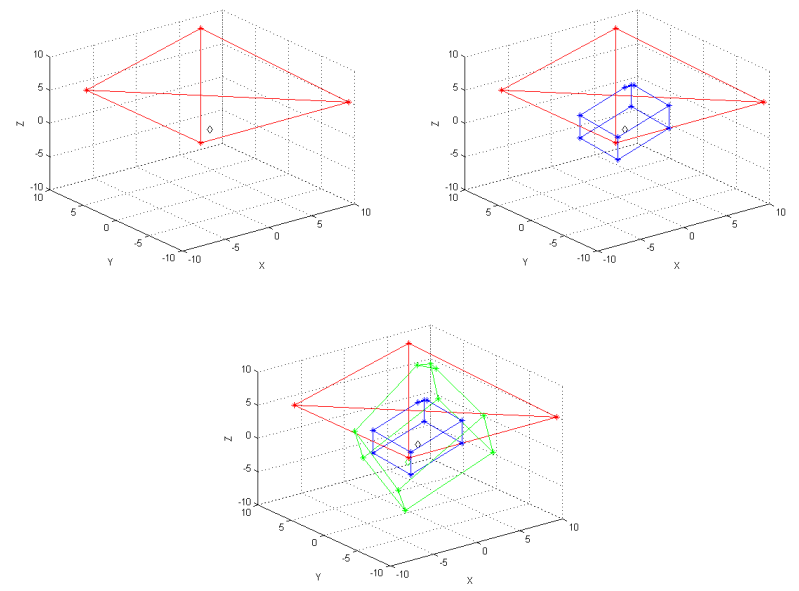

Figure 1. Prediction and expansion of target set

For maneuvering target tracking, this polyhedral description usually leads to a complex description as the number of vertices and supporting hyperplanes may become very high. This is why methods have been developed to approximate the complex polyhedron with a limited complexity over-bounding polyhedron Piet-Lahanier and Walter (1994). The modification consists in replacing the updated polyhedron by a surrounding polyhedron $\mathbf{P}$ whose number of vertices and supporting hyperplanes are limited a priori.

\section{COOPERATIVE MPC GUIDANCE}

\subsection{Cooperative cost definition}

The cooperative guidance laws must be designed for each of the $N$ interceptor missiles. The first step consists in defining the cooperative costs as presented in Section 2.1. As the mission consists in intercepting the target, a first component of the cost reflects the ability of the $i$ th missile to intercept the target. A classical cost function, translating the collision condition, is of the form

$$
g_{\text {inter }}^{i}=\left\|\mathbf{X}_{r} \times \mathbf{V}_{r}\right\|
$$

where $\mathbf{V}_{r}$ is the missile-target relative speed, $\mathbf{X}_{r}$ the missile target relative position and $\times$ the cross product. As the target speed is characterized under the form of a polyhedron, the corresponding cost is transformed as

$$
G_{\text {inter }}^{i}=\min _{\mathbf{x}^{c} \in \mathbf{P}} g_{\text {inter }}^{i}
$$

where $\mathbf{x}^{c}=\left[\left(\mathbf{X}^{c}\right)^{t},\left(\mathbf{V}^{c}\right)^{t}\right]^{t}$ is the target state vector (position and speed) and $\mathbf{P}$ is the surrounding limitedcomplexity polyhedral set. Let $\mathcal{V}^{i}=\arg \left(\min _{\mathbf{x}^{c} \in \mathbf{P}} g_{\text {inter }}^{i}\right)$ the vertex selected by missile $i$. In order to avoid competition among the interceptors, this vertex is removed from the description of $\mathbf{P}$. The cost (7) is thus modified as

$$
G_{\text {inter }}^{i}=\min _{\substack{\mathbf{x}^{c} \in \mathbf{P} \\ \mathbf{x}^{c} \notin\left\{\mathcal{V}^{j}, j=1, \ldots, N, j \neq i\right\}}} g_{\text {inter }}^{i}
$$

The cost function must be completed by other components reflecting the expected behavior of the missile raids. Limitation of energy translates directly in

$$
G_{\text {lim }}^{i}=\left\|\mathbf{u}^{i}\right\|
$$

Avoidance of other interceptor missiles is derived from costs defined in flocking behavior with collision avoidance and is expressed as

$$
\begin{array}{r}
d_{k}^{i, j}=\left\|\mathbf{x}_{k}^{i}-\mathbf{x}_{k}^{j}\right\|_{2}, \\
G_{\text {coll }}^{i}=\sum_{\substack{j=1 \\
j \neq i}}^{N} \sum_{k=1}^{H_{c}} \max \left(0, d_{\text {safe }}-d_{k}^{i, j}\right)
\end{array}
$$

where $d_{\text {safe }}$ is an a priori safety distance and $H_{c}$ is the control horizon. The cooperative guidance law is obtained by determining the optimal control sequence for each missile $i$ that minimizes the cost function consisting in the weighted sum of the previous cost functions:

$$
J^{i}=w_{\text {inter }} G_{\text {inter }}^{i}+w_{\text {lim }} G_{\text {lim }}^{i}+w_{\text {coll }} G_{\text {coll }}^{i} .
$$

Weight selection allows to put emphasis on some specific cost functions. After normalization, $w_{\text {inter }}$ is multiplied by 10 to stress the impact of the interception cost.

\subsection{Cooperation for multiple targets}

Extension to this approach to the case of $M$ targets is performed using the following approach. The prediction of all target states is performed as previously via boundederror approach, which provides $M$ polyhedrons. The optimal control sequence is obtained by first determining among these $M$ potential sets the reachable ones, given the limitation of each missile potential input range. The corresponding cost function is designed as

$$
G_{\text {mul }}^{i}=\min _{m=1, \ldots, M} \min _{\mathbf{x}^{c} \notin\left\{\mathcal{V}_{m}^{j}, j=1, \ldots, N, j \neq i\right\}} g_{\text {inter }}^{i}
$$

where $\mathbf{P}_{m}$ is the polyhedron associated with target $m$ and $\mathcal{V}_{m}^{i}$ is defined by $\mathcal{V}_{m}^{i}=\arg \left(\min _{\mathbf{x}^{c} \in \mathbf{P}_{m}} g_{\text {inter }}^{i}\right)$. In order to make this cost easier to compute, the evaluation of the 
cost is only performed on the extreme vertices of the $M$ polyhedron, i.e. the twelve vectors (at most) for which one component is equal to a minimal or a maximal value of the corresponding component of the state vector.

The global cost function for missile $i$ is

$$
J^{i}=w_{\text {inter }} G_{\text {mul }}^{i}+w_{\text {lim }} G_{\text {lim }}^{i}+w_{\text {coll }} G_{\text {coll }}^{i} .
$$

where the two costs $G_{l i m}^{i}$ and $G_{\text {coll }}^{i}$ are defined in equations (9) and (11). The weight selection procedure described in the previous paragraph has been used.

\subsection{Control computation}

The $i$ th missile movement is described by the following discrete dynamics:

$$
\left\{\begin{aligned}
x_{k+1}^{i} & =x_{k}^{i}+\tau v_{k}^{i} \cos \psi_{k}^{i} \cos \gamma_{k}^{i} \\
y_{k+1}^{i} & =y_{k}^{i}+\tau v_{k}^{i} \sin \psi_{k}^{i} \cos \gamma_{k}^{i} \\
z_{k+1}^{i} & =z_{k}^{i}+\tau v_{k}^{i} \sin \gamma_{k}^{i} \\
v_{k+1}^{i} & =v_{k}^{i} \\
\psi_{k+1}^{i} & =\psi_{k}^{i}+\tau u_{\psi, k}^{i} \\
\gamma_{k+1}^{i} & =\gamma_{k}^{i}+\tau u_{\gamma, k}^{i}
\end{aligned}\right.
$$

with $x^{i}, y^{i}, z^{i}$ the vehicle position components, $v^{i}$ its speed amplitude, $\psi^{i}$ and $\gamma^{i}$ its direction angles. The control inputs consist in discretized turn rate increments that must be selected among an a priori selection of values. The missile state and input vectors are thus

$$
\mathbf{x}^{i}=\left(\begin{array}{c}
x^{i} \\
y^{i} \\
z^{i} \\
v^{i} \\
\psi^{i} \\
\gamma^{i}
\end{array}\right) \text { and } \mathbf{u}^{i}=\left(\begin{array}{c}
u_{\psi}^{i} \\
u_{\gamma}^{i}
\end{array}\right)
$$

where $\mathbf{x}^{i}$ and $\mathbf{u}^{i}$ vary respectively in $\mathcal{X}_{i}$ and $\mathcal{U}_{i}$. Each missile computes its control inputs at each time step as a solution of an optimization problem over the future predicted trajectory. For tractability reasons, finite prediction and control horizons, respectively denoted $H_{p}$ and $H_{c}$ are used.

The future control inputs and the resulting state trajectories are denoted:

$$
\begin{aligned}
& \mathbf{U}^{i}=\left[\left(\mathbf{u}_{k}^{i}\right)^{t},\left(\mathbf{u}_{k+1}^{i}\right)^{t}, \ldots,\left(\mathbf{u}_{k+H_{c}-1}^{i}\right)^{t}\right]^{t}, \\
& \mathbf{X}^{i}=\left[\left(\mathbf{x}_{k+1}^{i}\right)^{t},\left(\mathbf{x}_{k+2}^{i}\right)^{t}, \ldots,\left(\mathbf{x}_{k+H_{p}}^{i}\right)^{t}\right]^{t} .
\end{aligned}
$$

As $H_{c}<H_{p}$, the control inputs are assumed to be 0 after $H_{c}$ steps. Once the optimal input sequences $\mathbf{U}^{i^{\star}}$ have been computed, each missile communicates its predicted trajectory to the rest of the fleet and applies the first entry $\mathbf{u}_{k}^{i \star}$. The optimization problem at time $k$ is:

$$
\begin{gathered}
\operatorname{minimise} J^{i}\left(\mathbf{U}^{i}, \mathbf{X}^{i}\right) \\
\text { over } \mathbf{U}^{i} \in \mathcal{U}_{i}^{H_{c}} \\
\text { subject to } \mathbf{X}^{i} \in \mathcal{X}_{i}^{H_{p}}
\end{gathered}
$$

\section{SIMULATION EXAMPLES}

Two scenarios are presented for evaluation of the resulting performances. The first one is dedicated to design guidance laws for missiles aiming at intercepting a single target with potential maneuvering abilities. The second one considers the interception of multiple targets.

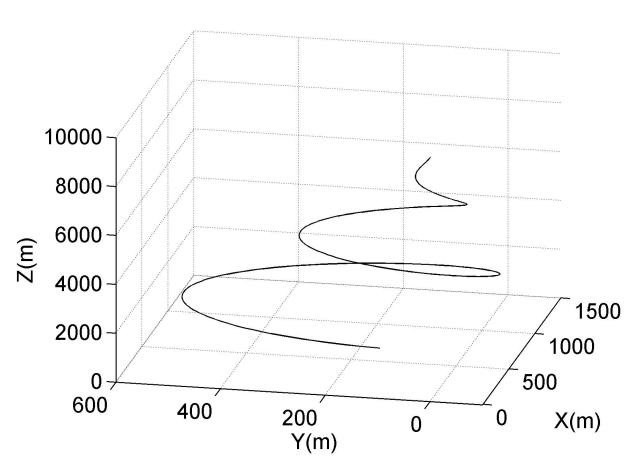

Figure 2. Target trajectory: Scenario 1

\subsection{Single target interception}

For illustrating the method described above, a maneuvering target trajectory has been defined as illustrated in Figure 2. For identification purposes, the target dynamics are modeled as

$$
\begin{aligned}
\mathbf{X}_{k+1}^{c} & =\mathbf{X}_{k}^{c}+\tau \mathbf{V}_{k}^{c} \\
\mathbf{V}_{k+1}^{c} & =\mathbf{V}_{k}^{c}+\tau \mathbf{u}_{k}^{c}
\end{aligned}
$$

where $\mathbf{X}^{c}$ and $\mathbf{V}^{c}$ are the position and speed vectors respectively, $\mathbf{u}^{\mathbf{c}}$ is the (unknown but bounded) input vector and $\tau$ is an a priori fixed parameter. Bounds on the input vector are set to $[-10 g, 10 g]$. The measurement vector $\mathbf{Z}_{k}^{c}$ at time $k$ consists in

$$
\mathbf{Z}^{c}(k)=\left(\begin{array}{c}
\mathbf{X}_{k}^{c}+\epsilon(k) \\
\mathbf{V}_{k}^{c}+\varpi(k)
\end{array}\right),
$$

where $\epsilon$ and $\varpi$ are uniformly distributed random vectors which vary respectively over the intervals $[-20 \mathrm{~m}, 20 \mathrm{~m}]$ and $\left[-50 \mathrm{~m} \cdot \mathrm{s}^{-1}, 50 \mathrm{~m} \cdot \mathrm{s}^{-1}\right]$. The measurement set at time $k$ is thus defined as

$$
\mathcal{Z}^{c}(k)=\left[\operatorname{Minz}_{k}, \mathbf{M a x z}_{k}\right]
$$

with

$$
\begin{aligned}
& \mathbf{M i n z}_{k}=\min \left(\left[\left(\mathbf{X}_{k}^{c}\right)^{t},\left(\mathbf{V}_{k}^{c}\right)^{t}\right]^{t}\right), \\
& \mathbf{M a x z}_{k}=\max \left(\left[\left(\mathbf{X}_{k}^{c}\right)^{t},\left(\mathbf{V}_{k}^{c}\right)^{t}\right]^{t}\right)
\end{aligned}
$$

where min and max define the respective minimal and maximal values of the measurement vectors (term by term), given the actual measurements and the assumed measurement bounds, $k$ evolves with a time step of 1 second. Figure 3 illustrates the polyhedron obtained after measurement updating projected in the position state space. Figures 4 and 5 present the resulting tracking. The estimated variation of target speed is constrained in a simple 3D polyhedron whose vertices are easily computed. Interception of this target is performed by two interceptor missiles, which are assumed to be located initially at an altitude $z=100 \mathrm{~m}$ and $x, y$ randomly chosen. The initial speed amplitude is $V_{0}=600 \mathrm{~m} \cdot \mathrm{s}^{-1}, \gamma$ is set equal to $45 \mathrm{deg}$ and $\psi$ is randomly chosen in [-90 deg, $90 \mathrm{deg}]$. The prediction and control horizons are respectively set to $H_{p}=10$ and $H_{c}=5$. The maximum number of vertices is equal to 12 .

Figure 6 illustrates the resulting trajectories obtained for the scenario. The black trajectories corresponds to the extreme estimated target trajectories. The interceptor missile trajectories are plotted in green and red. 


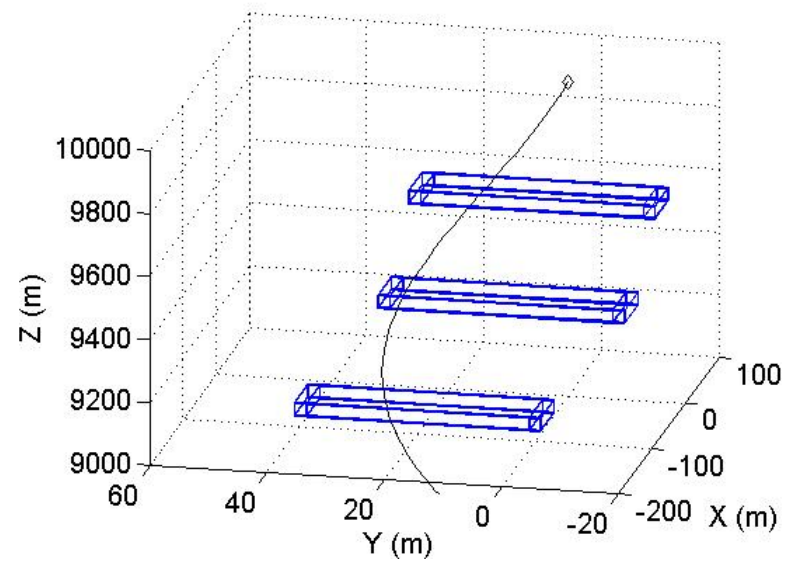

Figure 3. Estimation of target set in blue; Real target trajectory is black

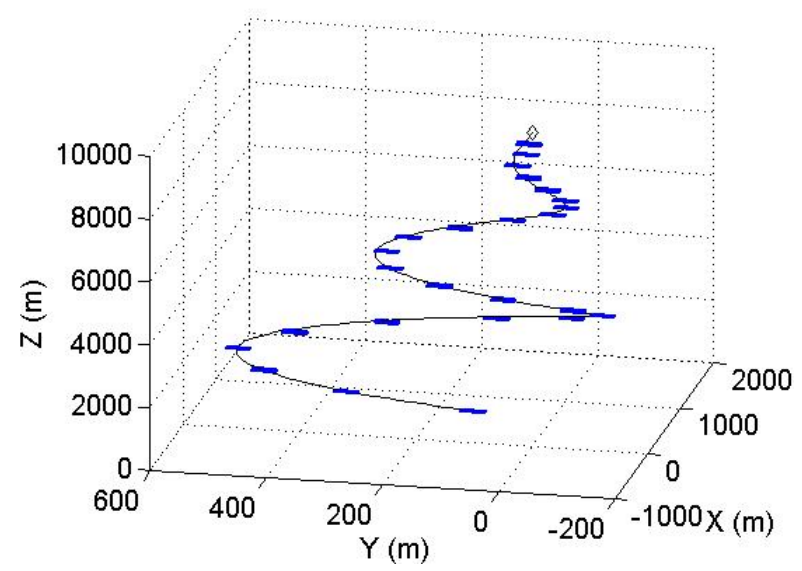

Figure 4. Estimation of target position in blue; Real target trajectory is black

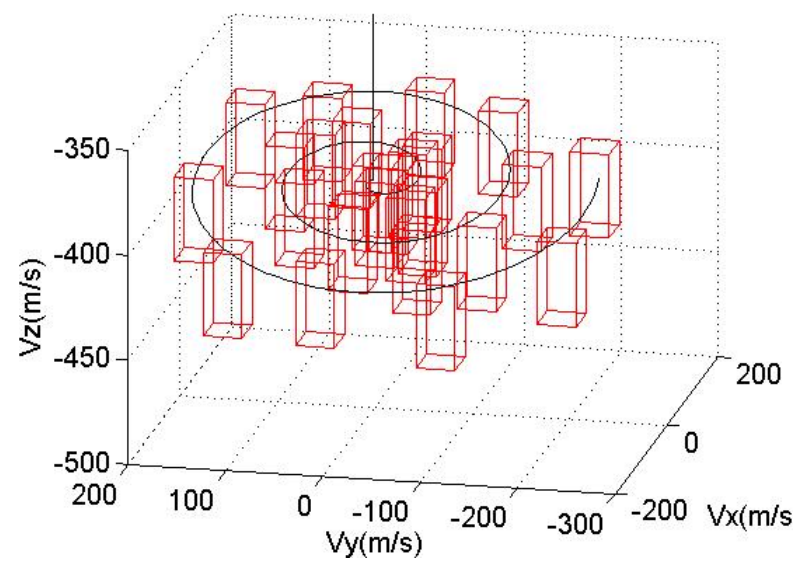

Figure 5. Estimation of target speed in red; Real target speed is black

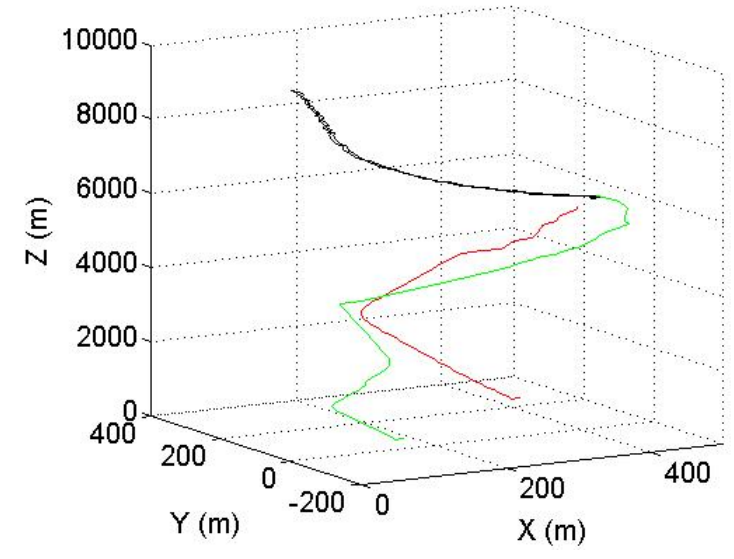

Figure 6. Cooperative interception for Scenario 1; Interceptors are in red and green

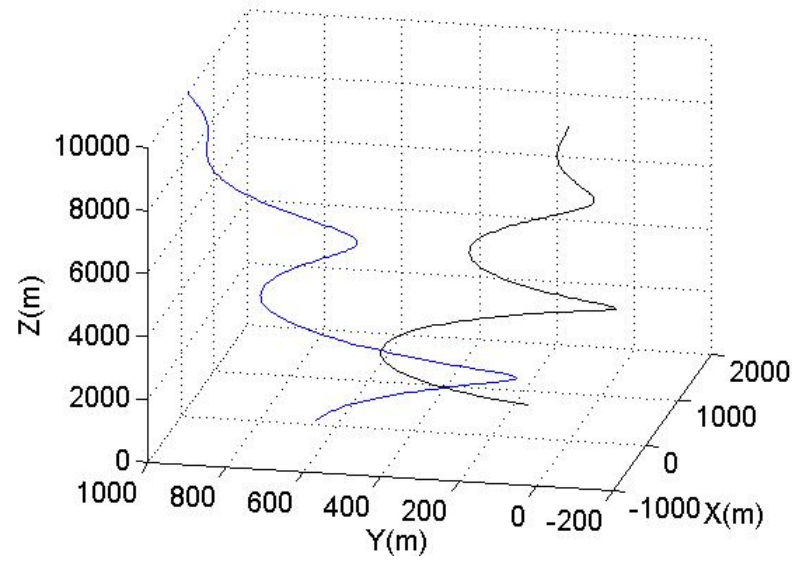

Figure 7. Trajectories of targets: Scenario 2

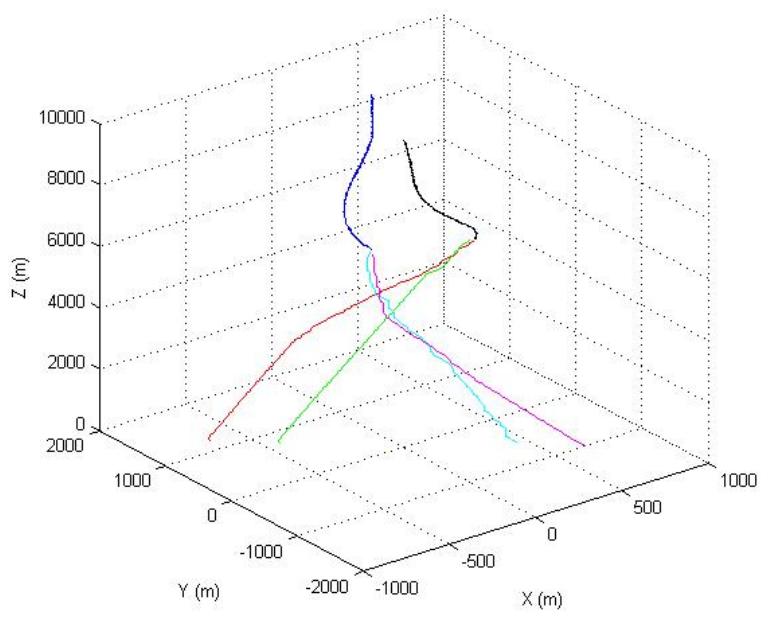

Figure 8. Cooperative interception for Scenario 2; Interceptors are in cyan, magenta, red and green 


\subsection{Multi-target interception}

In this scenario, two maneuvering targets are considered (Figure 7). Using (14), four interceptor missiles are guided towards the targets for interception. The resulting trajectories are illustrated in Figure 8 where the target trajectories are indicated in blue and black and the interceptor missile trajectories are in cyan, magenta, red and green.

\section{CONCLUSION}

In this paper, cooperative guidance laws for interception of maneuvering targets have been presented. They rely on the combination of polyhedral set description of the target location and speed variation and model predictive control design. Derivation of guidance cost expressions for single target and multi-target interception have been described. The first tests are promising, even if it remains to study the robustness of the approach to the available knowledge on the relative vehicle positions and speeds, as well as the ability of redefining the mission if information about potential vehicle failures must be taken into account.

\section{REFERENCES}

Ahmadzadeh, A., Keller, J., Pappas, G., Jadbabaie, A., and Kumar, V. (2006). An optimization-based approach to time critical cooperative surveillance and coverage with unmanned aerial vehicles. In Proceedings of the 10th International Symposium on Experimental Robotics, Rio de Janeiro, Brazil.

Bertsekas, D. and Rhodes, I. (1971). Recursive state estimation for a set-membership description of uncertainty. IEEE Transactions on Automatic Control, 16(2), 117128.

Best, R. and Norton, J. (2000). Predictive missile guidance. Journal of Guidance, Control, and Dynamics, 23(3), 539-546.

Chakravarthy, A. and Ghose, D. (2011). Collision cones for quadric surfaces. IEEE Trans. on Robotics, 27(6), 1159-1166.

Chandler, P., Pachter, M., and Rasmussen, S. (2001). UAV cooperative control. In Proceedings of the American Control Conference, Arlington, USA, 50-55.

Dunbar, W. and Murray, R. (2004). Receding horizon control of multi-vehicle formations: A distributed implementation. In Proceedings of the 43rd IEEE Conference on Decision and Control, Atlantis, Bahamas, volume 2, 1995-2002.

Durieu, C., Walter, E., and Polyak, B. (2001). Multiinput multi-output ellipsoidal state bounding. Journal of Optimization Theory and Applications, 111(2), 273303.

Garcia, C., Prett, D., and Morari, M. (1989). Model predictive control: theory and practicea survey. Automatica, 25(3), 335-348.

Gerkey, B. and Mataric, M. (2002). Sold!: Auction methods for multirobot coordination. IEEE Transactions on Robotics and Automation, 18(5), 758-768.

Gillen, D. and Jacques, D. (2002). Cooperative behavior schemes for improving the effectiveness of autonomous wide area search munitions. Cooperative control and optimization, 95-120.
Jadbabaie, A., Lin, J., and Morse, A. (2003). Coordination of groups of mobile autonomous agents using nearest neighbor rules. IEEE Transactions on Automatic Control, 48(6), 988-1001.

Le Menec, S., Markham, K., Tsourdos, A., Shin, H.S., and Piet-Lahanier, H. (2011). Cooperative allocation and guidance for air defence application. In Proceedings of the 18th IFAC World Congress, Milan, Italy, 3897-3902.

Müller, M., Reble, M., and Allgöwer, F. (2011). A general distributed mpc framework for cooperative control. In Proceedings of the 18th IFAC World Congress, Milan, Italy, 7987-7992.

Murray, R. (2007). Recent research in cooperative control of multivehicle systems. Journal of Dynamic Systems, Measurement, and Control, 129(5), 571.

Olfati-Saber, R., Dunbar, W., and Murray, R. (2003). Cooperative control of multi-vehicle systems using cost graphs and optimization. In Proceedings of the American Control Conference, Denver, Colorado, USA, volume 3, 2217-2222.

Piet-Lahanier, H. and Walter, E. (1994). Bounded-error tracking of time-varying parameters. IEEE Transactions on Automatic Control, 39(8), 1661-1664.

Rasmussen, S., Shima, T., Mitchell, J., Sparks, A., and Chandler, P. (2004). State-space search for improved autonomous uavs assignment algorithm. In IEEE Conference on Decision and Control, volume 3, 2911 - 2916.

Rochefort, Y., Bertrand, S., Piet-Lahanier, H., Beauvois, D., and Dumur, D. (2012). Cooperative non-linear model predictive control for flocks of vehicules. In Proceedings of IFAC Workshop on Embedded Guidance, Navigation and Control in Aerospace, Bangalore, India.

Rochefort, Y., Piet-Lahanier, H., Bertrand, S., Beauvois, D., and Dumur, D. (2011). Guidance of flocks of vehicles using virtual signposts. In Proceedings of the 18th IFAC World Congress, Milan, Italy, 5999-6004.

Schweppe, F. (1968). Recursive state estimation: unknown but bounded errors and system inputs. IEEE Transactions on Automatic Control, 13(1), 22-28.

Shin, H.S., Piet-Lahanier, H., Tsourdos, A., Le Menec, S., Markham, K., and White, B. (2011). Membership setbased mid course guidance: application to manoeuvreing target interception. In Proceedings of the 18th IFAC World Congress, Milan, Italy, 3897-3902.

Singer, R. and Behnke, K. (1971). Real-time tracking filter evaluation and selection for tactical applications. IEEE Transactions on Aerospace and Electronic Systems, 7(1), $100-110$.

Siva, E. and Maciejowski, J. (2011). Robust multiplexed MPC for distributed multi-agent systems. In Proceedings of the 18th World Congress, Milan, Italy, 251-256.

Walter, E. and Kieffer, M. (2003). Interval analysis for guaranteed nonlinear estimation. In Proceedings of the 13th IFAC Symposium on System Identification, Rotterdam, Netherlands, 259-270.

Walter, E. and Piet-Lahanier, H. (1989). Exact recursive polyhedral description of the feasible parameter set for bounded-error models. IEEE Transactions on Automatic Control, 34(8), 911-915.

Wei, M., Chen, G., Cruz, J., and Blasch, E. (2008). Multimissile interception integrating new guidance law and game theoretic resource management. In Proceedings of the IEEE Aerospace Conference, Big Sky, USA, 1-13. 